\title{
Translational Diffusion of Transient Intermediate Species during Protein Reactions: Phototropin
}

\author{
Masahide Terazima $^{1}$, Kimitoshi Takeda ${ }^{1}$, Yusuke Nakasone ${ }^{1}$, Kazunori Zikihara ${ }^{2}$, Satoru Tokutomi \\ ${ }^{1}$ Department of Chemistry, Graduate school of Science, Kyoto University \\ Kitashirakawa Oiwake-cho, Sakyo-ku, Kyoto 606-8502, Japan \\ mteazima@kuchem.kyoto-u.ac.jp \\ ${ }^{2}$ Department of Biological Science, Graduate school of Science, Osaka Prefecture University \\ 1-2 Gakuenmach, Sakai, Osaka 599-8531, Japan
}

\section{Extended Abstract}

Biological function involves a series of chemical reactions of biological molecules, and during these reactions, there are numerous spectrally silent dynamic events that cannot be monitored by absorption or emission spectroscopic techniques. Such spectrally silent dynamics include changes in conformation, intermolecular interactions (hydrogen bonding, hydrophobic interactions), inter-protein interactions (oligomer formation, dissociation reactions) and conformational fluctuations. These events are tightly associated with biological function. To understand the molecular mechanisms of reactions, time-resolved detection of such dynamics is essential. Recently, it has been shown that time-resolved detection of the translational diffusion is a powerful tool for measuring dynamic events. This technique is complementary to optical detection methods. For that purpose, we have developed a time-resolved detection of the translational diffusion coefficient in time-domain based on a pulsed laser induced transient grating (TG) technique [1,2]. Molecular translational diffusion is the process by which matter is transported from one part of position to another as a result of random molecular motions. The diffusion coefficient (D) reflect the diffusing molecular size. Hence, if we can monitor the D changes in time-domain, we can see how the molecular size changes by the reaction. More importantly, not only the size, but also the intermolecular interaction affects the magnitude of $\mathrm{D}$. If the friction between the diffusing solute and the solvent molecules increases, $\mathrm{D}$ should decrease. Therefore, measurement of $\mathrm{D}$ in time-domain would be useful and powerful technique to study the time development of not only the molecular size but also intermolecular interaction, both of which are spectrally silent.

By using this diffusion detection method, we have studied variety of reactions of proteins, such as a blue light sensor protein phototropin, which controls the phototropism of a plant. We will present results of reactions of LOV2 (Light-OxygenVoltage 2) domain of phototropin. Recently, conformational changes of the amino-terminal helix (A' $\alpha$ helix), in addition to the reported conformational changes of the carboxyl-terminal helix ( $\mathrm{J} \alpha$ helix), have been proposed to be important for the regulatory function of LOV2 of phototropin 1 from Arabidopsis. However, the reaction dynamics of the A' $\alpha$ helix have not been examined. Here, the unfolding reactions of the $\mathrm{A}^{\prime} \alpha$ and $J \alpha$ helices of the LOV2 domain of phototropin 1 were investigated by the diffusion detection method. A mutant (T469I mutant) that renders the A' $\alpha$ helix unfolded in the dark state showed unfolding of the J $\alpha$ helix with a time constant of $1 \mathrm{~ms}$, which is very similar to the time constant reported for the wild-type LOV2-linker sample. Furthermore, a mutant (I608E mutant) that renders the J $\alpha$ helix unfolded in the dark state exhibited an unfolding process of the $\mathrm{A}^{\prime} \alpha$ helix with a time constant of $12 \mathrm{~ms}$. On the basis of these experimental results, it is suggested that the unfolding reactions of these helices occurs independently.

\section{References}

[1] M. Terazima, "Time-Dependent Intermolecular Interaction during Protein Reactions," Phys. Chem. Chem. Phys., vol. 13, pp. 16928-16940, 2011.

[2] M. Terazima, "Studies of photo-induced protein reactions by spectrally silent reaction dynamics detection methods: Applications to the photoreaction of the LOV2 domain of Phototropin from Arabidopsis Thaliana," Biochim. Biophys. Acta, vol. 1814, pp. 1093-1105, 2011. 